\title{
MÉTODO DE PLANIFICACIÓN DE TRAYECTORIAS MÚLTIPLES PARA ENJAMBRE DE UAVS.
}

\author{
Ángel Madridano, Abdulla Al-Kaff, David Martín, Arturo de la Escalera, y J.M. Armingol \\ Laboratorio de Sistemas Inteligentes (LSI), Universidad Carlos III de Madrid. \\ Avda. de la Universidad 30, 28911, Leganés, Madrid, España \\ \{amadrida, akaff, dmgomez, escalera, armingol\}@ing.uc3m.es.
}

\section{Resumen}

Los avances en robótica han provocado la aparición del uso cooperativo y autónomo de distintos tipos de vehículos, lo que permite completar de manera más eficiente diferentes tipos de trabajos. Uno de los aspectos fundamentales relacionados con esta colaboración es la planificación de rutas seguras, de tal manera, que se pueda garantizar el movimiento libre de colisiones de cada uno de los vehículos por el área de trabajo. Por este motivo, se propone una planificación de rutas múltiples para un enjambre de Vehículos Aéreos No tripulados basado en la creación de Hojas de Rutas Probabilísticas. El objetivo del trabajo es la implementación de un método para la construcción de trayectorias seguras para cada uno de los agentes del enjambre en diversas situaciones, tales como: alcanzar un único objetivo de manera conjunta, o dividirse para alcanzar diferentes ubicaciones con los de diversos vehículos que conforman el enjambre (caso etiquetado y no etiquetado). Para ello, se empleará Hojas de Rutas Probabilísticas, demostrando que aportan una solución eficiente al problema de planificación de múltiples rutas. Además, se propone la integración de esta solución en una arquitectura en $R O S$ que permita, en primera instancia obtener resultados dentro del entorno de simulación Gazebo y, en un futuro, una sencilla implementación de los algoritmos presentados en una flota de vehículos real. Los resultados obtenidos muestran un rendimiento óptimo en cuanto a tiempo de cómputo para las distintas situaciones propuestas y, la posible integración en un framework versátil como ROS.

Palabras clave: UAVs · Planificación · Enjambres · Autónomo · Navegación · ROS.

\section{Introducción}

Los desarrollos en el área de la electrónica han provocado la mejora de la versatilidad y aplicabilidad de los sistemas móviles robóticos. La reducción del peso y coste de los dispositivos instalados en los diferentes vehículos y el aumento de su autonomía ha provocado la irrupción de los
Vehículos Aéreos No Tripulados (UAVs) y de los Vehículos Terrestres No Tripulados (UGVs) en un gran número de actividades militares [21] y civiles, como la inspección de infraestructuras [2], monitorización del tráfico[7], entregas[3] y labores de búsqueda y rescate[1].

La posibilidad de complementar las diferentes características de los distintos tipos de vehículos ha provocado que en los últimos años se extienda el uso de los Sistemas Multi-Robot (MRS) para acometer una amplia variedad de tareas tanto en entornos estructurados y seguros como en ambientes abiertos, dinámicos y hostiles. Uno de los aspectos más importantes a tratar dentro de MRS es la planificación [6], definida como la tarea de crear una secuencia de acciones que permita a los diferentes vehículos alcanzar una meta[15]. Este aspecto incluye dos fases distintas: planificación de tareas, donde el robot debe ejecutar cada tarea, y planificación de movimiento, que busca generar una trayectoria segura para cada vehículo[19].

Los métodos para la planificación de trayectorias permiten dotar a los MRS de autonomía a la hora de acometer misiones, además de, garantizar la seguridad de todos los elementos presentes en el entorno al planificar las rutas considerando colisiones tanto con los obstáculos estáticos como con los dinámicos presentes en el entorno. En este trabajo se presenta un método de planificación de rutas para MRS en diferentes situaciones y aplicable en 2D y $3 \mathrm{D}$. El desarrollo realizado puede orientarse tanto UGVs como UAVs, pero el enfoque final es la planificación de trayectorias para una flota de drones que alcance diferentes objetivos en distintas situaciones. La idea principal es desarrollar un método basado en hojas de rutas probabilísticas como solución al problema de establecer múltiples trayectorias para múltiples objetivos dentro de un MRS.

Este trabajo se organiza de la siguiente manera: sección 2 presenta una revisión de la literatura relacionado con los trabajos recientes en el campo de la planificación de trayectoria para MRS. A continuación, la Sección 3 describe el método de planificación de trayectorias propuesto para alcanzar los objetivos en casos etiquetados, no eti- 
quetados o en aquellas situaciones en las que todos los vehículos deben alcanzar un solo objetivo. Una vez detallado nuestro trabajo, la Sección 4 recoge la implementación de los algoritmos en un entorno de simulación como Gazebo junto con un análisis de los obtenidos en las diferentes situaciones. Por último, la Sección 5 permite establecer las conclusiones y detallar los posibles desarrollos a realizar en el futuro.

\section{Estado del Arte}

Actualmente, existe una amplia gama de tecnologías disponibles para resolver el problema de la planificación de trayectorias, tales como métodos basados en gráficos, métodos basados en campos potenciales, métodos probabilísticos o métodos basados en el aprendizaje profundo. Dentro de las distintas formas de abordar este problema, los métodos probabilísticos permiten explorar grandes espacios sin aumentar exponencialmente el tiempo de cálculo y, además, en el caso de la planificación MRS, permiten reutilizar el gráfico generado tras la exploración del entorno tantas veces como agentes forman el sistema, amortizando así el gasto computacional derivado del uso de este tipo de métodos.

Los métodos probabilísticos agrupan dos grandes métodos de planificación, clasificados como pasivos y activos, dependiendo de si es necesario utilizar un segundo tipo de algoritmo para establecer el camino óptimo entre la configuración inicial y final [20]. Los métodos pasivos incluyen las Hojas de Ruta Probabilísticas (PRM), mientras que los métodos activos incluyen la Exploración Rápida de Árboles Aleatorios (RRT)[10].

En [4], se utiliza un planificador 2D basado en PRM orientado a que formaciones de UAV atraviesen áreas ricas en obstáculos. Otros trabajos, establecen un método en dos fases para poder establecer rutas óptimas para UAVs en terrenos complejos; en una primera fase, se emplea un algoritmo PRM para explorar el entorno y, a continuación, el algoritmo $\mathrm{D}^{*}$ lite para poder encontrar las rutas óptimas [17].

En [11], se presenta un PRM mejorado que considera la información del terreno y las posibles amenazas para generar la hoja de ruta, construyendo trayectorias seguras en 3D. En otros trabajos, como por ejemplo [18], se utiliza la información de los algoritmos de Octree para realizar un PRM en $3 \mathrm{D}$. Octree es una estructura jerárquica de datos que subdivide el espacio 3D en volúmenes cúbicos llamados vóxeles, que se subdividen hasta alcanzar una resolución esperada. Cuando se crean, se determina si este volumen está libre u ocupado y, con esta información, se emplea un PRM para la exploración del entorno y, finalmente, establecer trayectorias libres de colisión utilizando el algoritmo A*[10]. Siguiendo esta línea de investigación, [16] presenta un trabajo en el que utiliza métodos de mejora híbridos o de PRM para planificar rutas $3 \mathrm{D}$ para UAVs.

Por último, están empezando a aparecer métodos que permiten a los UAV navegar con seguridad a través de un aprendizaje reforzado y un aprendizaje en profundidad. Sin ir más lejos, [5] presenta una hibridación de PRM con Aprendizaje Reforzado para establecer trayectorias en entornos urbanos para tareas de entrega con UAVs.

Aunque los métodos propuestos en este trabajo se realizan en una exploración $2 \mathrm{D}$, la ruta generada para los diferentes UAVs, considera la posibilidad de volar a diferentes alturas, para evitar colisiones entre drones, por lo que las trayectorias generadas son en 3D. En este trabajo se utilizan métodos pasivos, construyendo un PRM una sola vez y, utilizando el grafo obtenido tantas veces como agentes se tenga y, amortizando el coste computacional del mismo.

\section{Propósito de Planificación de trayectorias múltiples basado en PRM.}

Este trabajo se centra en el desarrollo de un método basado en PRM, que permita establecer la combinación de trayectorias óptimas para un sistema Multi-Robot que trabaja en un entorno estructurado. La idea es demostrar que estos métodos generan soluciones eficaces para este tipo de problemas y, sentar las bases para futuros desarrollos con exploraciones en 3D. Además, de implementar una arquitectura del métodos de planificación propuesto sobre el framework Robot Operating System (ROS) [14], permitiendo realizar las primeras simulaciones sobre Gazebo [8].

El trabajo presenta una solución para la planificación de trayectorias para MRS, en la que los diferentes agentes parten de múltiples posiciones de inicio y deben alcanzar una ubicación determinada recorriendo el espacio de trabajo de manera segura. Dentro de la solución implementada se establece un parámetro que permite definir tres casos o modos de planificación distintos:

1. Único Objetivo: todos los vehículos deben alcanzar una misma ubicación, por lo que el método generará como salida la ruta óptima para que cada uno de los UAVs llegue al objetivo marcado. Su aplicación final puede orientarse a formación en escenarios de emergen- 
cias como incendios o rescates.

2. Caso Etiquetado: se corresponde con aquellos problemas en los que cada uno de los vehículos tiene asignado por defecto un objetivo o posición final, es decir, se ha de calcular la trayectoria óptima para un caso simple de inicio o final tantas veces como agentes se tengan.

3. Caso No Etiquetado: es el más complejo de los presentados en el trabajo y, por tanto, requiere que se añada al método una fase final destinada a establecer la combinación de rutas óptimas que genere la mejor solución del problema. En este caso, se posee un mismo número de objetivos que de UAVs, y, se debe establecer que combinación de trayectorias óptima minimiza la distancia recorrida por el sistema.

Aunque dentro de los 3 diferentes casos de estudio existen diferencias a la hora de abordar la fase de selección de rutas óptimas, es cierto que ambos comparten un esquema general, formado por un conjunto de procedimientos comunes.

1. Generación de mapa de entorno: en esta primera propuesta, la tarea de planificación se realiza sobre una imagen binaria predefinida del entorno (Figura 1). Este tipo de mapa se caracteriza por tener el espacio libre en blanco y los obstáculos en negro.

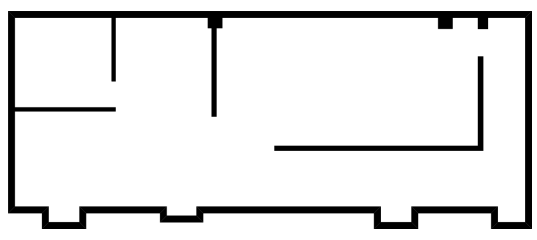

Figura 1: Mapa Inicial.

Además, se realiza un procedimiento de expansión de los bordes de los obstáculos (Figura 2), con el objetivo de dotar a la planificación de un margen de seguridad entorno a los mismos, cuyo valor está relacionado con el agente de mayor tamaño empleado. De esta manera, se asegura que todos los nodos creados en el espacio libre constituyen waypoints seguros de cara a la elaboración final de las rutas. Por último, en esta fase se añaden los puntos de partida de los agentes del MRS y los objetivos a alcanzar.

2. Exploración del entorno: para poder establecer una planificación de rutas es necesario realizar una exploración completa del área de trabajo. En este punto se ha decido

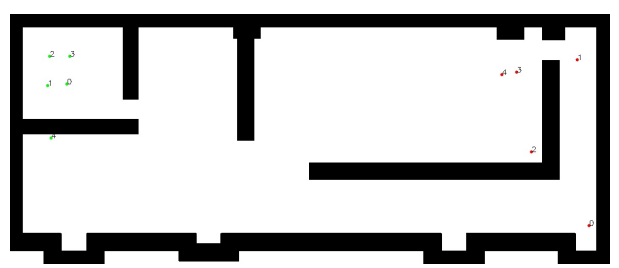

Figura 2: Mapa con extensión de obstáculos.

implementar un algoritmo basado en PRM. La principal razón para emplear este método, frente a otros algoritmos presentes en la literatura, es la posibilidad de reutilizar el grafo creado para los distintos vehículos, por lo que el coste de implementar este algoritmo se amortiza a ser empleado tantas veces como agentes forman el MRS.

El algoritmo propuesto se compone de un conjunto de fases claves para su correcta implementación:

- Adición de puntos iniciales y finales a los nodos: en una primera implementación la posición de partida de los agentes del MRS serán generadas por el usuario en un archivo TXT, junto con las posiciones objetivo a alcanzar. Dichos archivos serán leídos y, se comprobarán que todas ellas corresponden a espacio libre de obstáculos en el mapa. En caso afirmativo, todos los puntos serán añadidos a los nodos que formarán el grafo del PRM.

- Generación aleatoria de nodos: el número de nodos creados para explorar el entorno viene determinado por el usuario. Las coordenadas de cada nuevo nodo generado se establecen de manera aleatoria (Ecuaciones 1 y 2):

$$
\begin{gathered}
X=X_{\min }+\frac{\left(X_{\max }-X_{\min }\right) * \operatorname{rand}()}{R A N D_{-} M A X+1,0} \\
Y=Y_{\min }+\frac{\left(Y_{\max }-Y_{\min }\right) * \operatorname{rand}()}{R A N D_{-} M A X+1,0}
\end{gathered}
$$

donde RAND_MAX corresponde a una Macro constante de $\mathrm{c}++$ que retorna el máximo valor de $\operatorname{rand}()$, y el resto de variables son definidas por el usuario en función del tamaño del mapa. Una vez se ha generado el nodo, se comprueba que se encuentra dentro del espacio libre del entorno, es decir, que corresponde a un píxel blanco en la imagen del área de trabajo. Tras esta comprobación, se añade 
o descarta el nodo y se repite el proceso hasta tener en nuestro PRM el número de nodos determinados por el usuario.

- Generación de bordes: una vez se han añadido todos los nodos, el siguiente paso es establecer rutas libres de obstáculos entre dichos nodos. De cara a mejorar la eficacia del algoritmo, se establece un rango de distancias en las que el camino hallado entre dos nodos es válido. De esta manera, se evitará añadir dos nodos que se encuentren muy próximos y que no aporten una solución interesante. Estos rangos de distancia se establecen como una proporción del tamaño total del mapa del entorno, manteniendo la relación de distancias aunque el mapa a explorar cambie.

Por tanto, en esta fase se recorren todos los nodos creados a la vez que se calcula la distancia euclídea al resto de nodos. En el caso de que el valor de distancia obtenido se encuentre dentro del rango óptimo, se pasa a comprobar que dicha ruta se encuentra libre de obstáculo, comprobando que todos los puntos que forman la recta se encuentran en el espacio libre.

Finalmente, si el borde que une ambos nodos está libre de obstáculos se añade al grafo que conformará la hoja de ruta (Figura 3), que se empleará como entrada para el algoritmo de búsqueda de rutas óptimas. En relación a esta fase, es importante detallar que se ha configurado el algoritmo de tal manera que se evita que dos nodos correspondientes a puntos iniciales o finales puedan conectarse entre sí, para reducir la probabilidad de impacto entre diferentes UAVs.

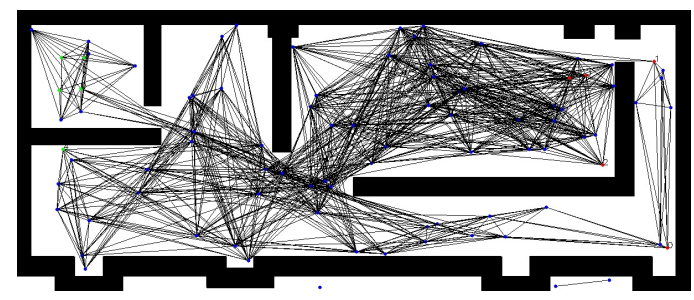

Figura 3: Mapa explorado con PRM de 80 nodos.

3. Generación de rutas: una vez generado el $\mathrm{PRM}$, es necesario hacer uso de algoritmos que permitan obtener la ruta óptima para cada uno de los casos. En este caso, se ha optado por emplear el algoritmo A* para encontrar la manera más eficiente de alcanzar los diferentes objetivos, estableciendo un procedimiento común para las 3 situaciones estudiadas. Aunque cada uno de los casos estudiados presenta particularidades a la hora de generar una solución óptima al problema de la planificación de rutas múltiples. Por lo que, a continuación, se describirá la propuesta realizada para cada una de las situaciones.

\subsection{Objetivo único.}

En este caso, el algoritmo A* se encarga de establecer una ruta para cada uno de los UAVS hacia el objetivo determinado por el usuario. A través del grafo generado y, considerando en cada caso el punto final y de partida, el algoritmo establece la ruta óptima en función de los valores de distancia euclídea de los bordes. De esta manera, en cada ruta seleccionada se ha optado por minimizar la distancia recorrida por cada UAV, para de esta manera generar una solución segura y eficaz que permita a los diferentes vehículos alcanzar un mismo destino (Figura 4).

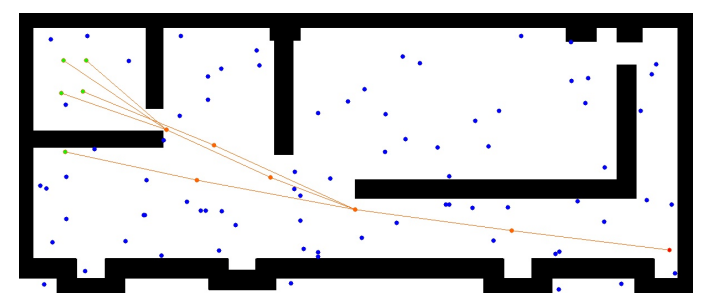

Figura 4: Solución Objetivo único (80 nodos).

Como sucede en el resto de los casos, una vez obtenidos el conjunto de waypoints en el mapa del entorno, se trasladan dichos puntos a coordenadas locales con respecto la posición inicial del UAV y se almacenan para ser empleados posteriormente dentro de la arquitectura de ROS. A la hora de determinar la altura o coordenada $\mathrm{z}$ de cada uno de los waypoints, en esta primera propuesta, se ha decidido dotar a cada UAV de una altura diferente, de tal manera que, si ambas rutas coinciden en algún punto, cada uno llegará con una altura suficiente para evitar la colisión entre ambos.

\subsection{Caso etiquetado.}

Es una extensión del caso anterior, dado que a cada UAV se le asigna un objetivo concreto. En este caso, se repite la metodología de la situación anterior para cada uno de los UAVs y para cada punto objetivo prefijado. De nuevo, el uso del algoritmo $A^{*}$ permite obtener, de manera rápida, una solución eficiente al problema de la planificación múltiple para cada vehículo(Figura 5). 


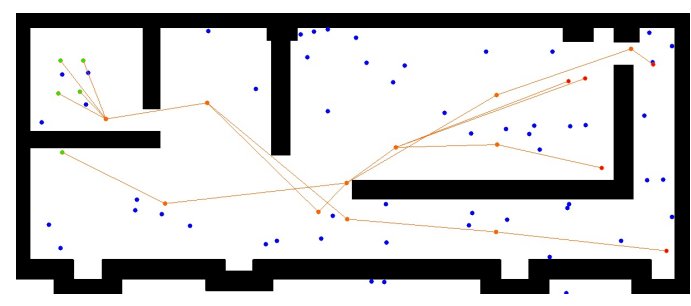

Figura 5: Solución Caso Etiquetado (65 nodos).

\subsection{Caso no Etiquetado.}

Es el caso de estudio más complejo, ya que requiere encontrar la combinación de inicios finales más eficiente y óptima. Para este caso, todas las rutas posibles entre los diferentes UAVS y los objetivos son calculadas, generando como solución tantas rutas como vehículos se dispongan de tal manera que la distancia conjunta recorrida sea la menor.

En común con los dos anteriores casos está el uso del algoritmo $A^{*}$ para determinar todas las rutas posibles entre cada UAV y los diferentes objetivos. De esta manera, se almacenarán todas las soluciones posibles obteniendo una matriz en la que para cada dron se tenga la distancia más corta a cada uno de los objetivos.

Una vez completado este paso, la siguiente tarea es implementar un método que permita obtener aquella combinación de rutas que garanticen alcanzar todos los objetivos y, minimice la distancia recorrida por el MRS. Para ello, se ha optado por implementar el método húngaro [9] que permite minimizar problemas de asignación como el que se genera en esta situación y, el cual ha sido empleado por otros investigadores en este campo [12]. De esta manera, implementando este método, se aporta una solución final con la planificación de trayectorias óptima para un caso no etiquetado(Figura 6).

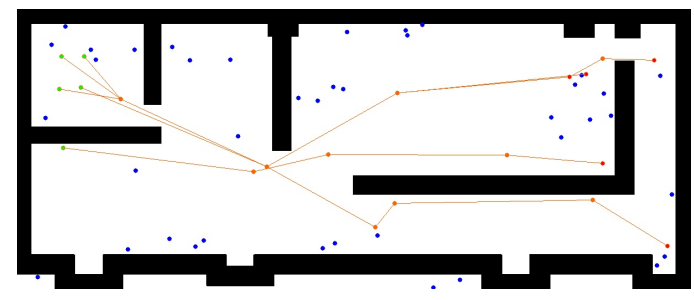

Figura 6: Solución Caso No Etiquetado (50 nodos).

\section{Simulación y Resultados.}

Los objetivos de este trabajo están orientados a establecer un método de planificación de múltiples rutas para múltiples vehículos basado en Hojas de Rutas Probabilísticas que aporte una solución óptima y eficaz al problema de la generación de trayectorias en MRS y, a integrar este método de planificación en una Arquitectura en ROS, de tal forma que permita una implementación futura de los algoritmos en una flota de UAVs real.

En lo referente a la integración del método en ROS, se ha llevado a cabo un desarrollo que permite implementar la solución a la planificación de trayectorias dentro del entorno de simulación de Gazebo, empleando conjuntamente el paquete hector_quadrotor [13]. Para ello ha sido necesario recrear el entorno estructurado sobre el que se ha trabajado en la planificación y, desarrollar un método encargado de leer y publicar las rutas de cada una de las aeronaves. Además, dicho desarrollo será el encargado de establecer intercambios de comunicación con los UAVs para conocer su posición actual, verificar que se alcanzan las posiciones intermedias fijadas y, asegurarse de que el objetivo es alcanzado y, por tanto, la ruta es completada. En la Figura 7, se puede observar como los UAVs alcanzan las posiciones objetivos correspondientes al entorno y caso no etiquetado mostrado en la Figura 6 completando las rutas generados por el método propuesto.

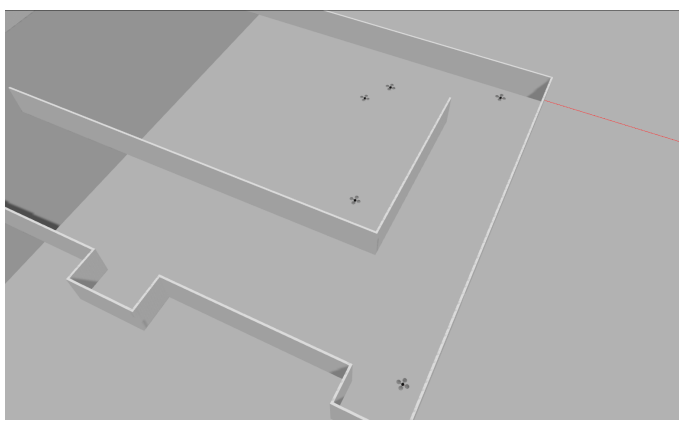

Figura 7: Solución del Caso No Etiquetado mostrado en la Figura 6 simulado en Gazebo.

La propuesta de PRM para múltiples objetivos dentro de una flota de UAVs ha sido implementada satisfactoriamente en Gazebo ${ }^{1}$, realizando simulaciones para los diferentes casos y objetivos, comprobando que en todas las pruebas los vehículos son capaces de alcanzar su posición final sin colisionar con los obstáculos estáticos presentes en el entorno o con los objetos dinámicos correspondientes al resto de vehículos en movimiento (Figura 8). Es importante destacar que, a la hora de im-

\footnotetext{
${ }^{1}$ Para más detalles, ver video: https://youtu.be/RAyP0Ah9Phs
} 
plementar este método en entornos estructurados como el del ejemplo, es necesario considerar una alta precisión a la hora de alcanzar un waypoint intermedio de la ruta. Ya que, en el caso de aceptar un error de posición entorno superior al metro, se pueden producir colisiones con el entorno por acumular errores en las trayectorias. Además, es necesario un ajuste fino de los parámetros del controlador PID incluido en el paquete hector_quadrotor para poder alcanzar las posiciones correctamente.

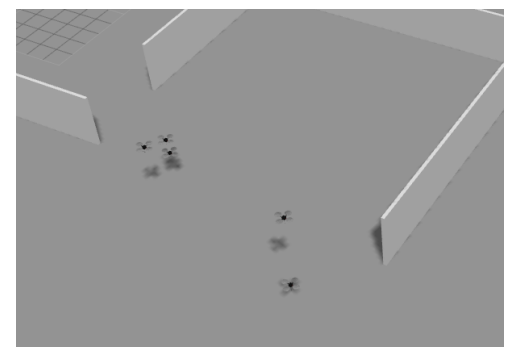

Figura 8: MRS in a structured environment.

En lo que respecta a la generación de hojas de rutas probabilísticas para resolver el problema de la multi-planificación de rutas en MRS, a vista de los resultados obtenidos se puede determinar que su implementación y desarrollo aporta una solución eficaz al problema de la planificación de trayectorias.

Cuadro 1: Comparación del tiempo de cómputo en función del número de UAVs y de las diferentes situaciones para 80 nodos.

\begin{tabular}{|c|c|c|c|}
\hline & \multicolumn{3}{|c|}{ Tiempo de Cómputo (s) } \\
\hline Número de UAVs & Único Objetivo & Caso Etiquetado & Caso No Etiquetado \\
\hline 5 & 0.0334 & 0.0334 & 0.0379 \\
\hline 8 & 0.0378 & 0.0383 & 0.0503 \\
\hline 10 & 0.0405 & 0.0416 & 0.0547 \\
\hline 15 & 0.0460 & 0.0469 & 0.0905 \\
\hline
\end{tabular}

La principal razón para usar este método de planificación estaba ligada a la idea de reutilizar la hoja de ruta generada para los diferentes vehículos de nuestro MRS, y de esta manera, amortizar el tiempo de cómputo.

En la Tabla 1 se recoge el tiempo de cómputo de nuestro método en función del número de UAVs que formen el MRS para las diferentes situaciones, con una exploración constante de 80 nodos. El motivo de usar este valor constante de nodos y no otro, es que, para todas las situaciones, este valor garantiza que la exploración será suficientemente buena como para encontrar una solución. Como se puede observar, para el caso de un único objetivo y el caso etiquetado el tiempo de cómputo apenas crece al aumentar el número de vehículos que forman el MRS, lo cual justifica el uso del método desarrollado basado en PRM para la planificación de múltiples. En el caso no etiquetado, si se produce una variación a medida que el MRS crece, pero dicho aumento está relacionado con la ejecución del método húngaro para optimizar la asignación de rutas y, con la creación del PRM como base para encontrar rutas óptimas.

Analizando los resultados de la Tabla 2, en los cuales se recoge el tiempo de cómputo empleado en encontrar una solución óptima en función del número de nodos para cada uno de los casos estudiados. El primer apunte es que el tiempo reflejado es el tiempo medio obtenido tras 20 ejecuciones del mismo caso, lo cuál permite obtener un valor más fiable y real y, además, permite obtener información sobre cual es el mínimo número de nodos necesario para obtener una solución en todos los casos. Así pues, tras ejecutar en 20 ocasiones cada una de las situaciones recogidas en la Tabla 2, para 80 nodos, siempre se ha obtenido una solución completa. Indicar también, que para estos casos se ha trabajado con un número fijo de UAVs y de objetivos finales iguales a 5 .

En el caso del objetivo único, se comprobó que, para los diferentes objetivos propuestos, el tiempo de cómputo era similar, es decir, llegar a un punto $\mathrm{u}$ otro no afecta al gasto computacional realizado, por lo que se han incluido únicamente los datos en los que todos los agentes del sistema acuden al primero de los objetivos.

\section{Conclusiones y Trabajos Futuros}

La planificación de trayectorias para MRS se ha convertido en uno de los campos en desarrollo de la robótica debido a la importancia de poder generar trayectorias óptimas y seguras en diferentes áreas de trabajo. Por esta razón, el presente documento propone un método de planificación basado en PMR de trayectorias múltiples, de modo que los distintos vehículos aéreos no tripulados alcancen objetivos diferentes. Además, el método se prueba en 3 casos de uso diferentes: todos los agentes a un solo objetivo, cada agente alcanza un objetivo previamente establecido o, cada uno de los vehículos en el sistema va a un objetivo de tal manera que la distancia total recorrida por el MRS se minimiza.

Los resultados recogidos de la propuesta demuestran que la generación de PRM para la planificación de múltiples rutas en MRS está justificada dado que el gasto computacional derivado de la creación de esta se amortiza con el aumento de vehículos y, por tanto, constituye una herramienta eficaz para resolver problemas de planificación de trayectorias en sistemas escalables. Además, se presenta un método capaz de generar soluciones óptimas para diferentes situaciones dentro de un entorno estructurado, el cual se ha demostrado, a 
Cuadro 2: Comparación del tiempo de cómputo en función del número de nodos y de la situación propuesta.

\begin{tabular}{|c|c|c|c|c|c|c|c|c|c|c|c|c|c|c|c|}
\hline \multicolumn{16}{|c|}{ Tiempo de Cómputo (s) del Algoritmo PRM para cada caso de estudio. } \\
\hline nNodes & 10 & 20 & 30 & 40 & 50 & 60 & 70 & 80 & 90 & 100 & 110 & 120 & 130 & 140 & 150 \\
\hline Único Objetivo & 0.0210 & 0.0218 & 0.0233 & 0.0246 & 0.0261 & 0.0287 & 0.0311 & 0.0334 & 0.0371 & 0.0415 & 0.0444 & 0.0486 & 0.0539 & 0.0582 & 0.0641 \\
\hline Caso Etiquetado & 0.0213 & 0.0222 & 0.0229 & 0.0248 & 0.0260 & 0.0280 & 0.0311 & 0.0334 & 0.0366 & 0.0401 & 0.0437 & 0.0498 & 0.0539 & 0.0562 & 0.0631 \\
\hline Caso No Etiquetado & 0.0209 & 0.0223 & 0.0238 & 0.0256 & 0.0284 & 0.0308 & 0.0334 & 0.0379 & 0.0420 & 0.0460 & 0.0514 & 0.0591 & 0.0659 & 0.0704 & 0.0789 \\
\hline
\end{tabular}

través de simulaciones en Gazbeo, que puede ser integrado en ROS como paso previo a su implementación en un MRS real.

Finalmente, la investigación futura se centra en la exploración del entorno completo en 3D, y en el estudio de métodos alternativos al método húngaro, que pueden mejorar el gasto computacional del caso no etiquetado al aumentar el número de agentes en el MRS.

\section{Agradecimientos}

Este trabajo es soportado por el gobierno de la Comunidad de Madrid a través de la Beca Doctorado Industrial (BECA IND2017/TIC-7834).

Este trabajo fue financiado por el Gobierno de España a través de dos proyectos CICYT (TRA201678886-C3-1-R, RTI2018-096036-B-C21), la Comunidad de Madrid con P2018/EMT-4362).

\section{English summary}

\section{MULTI-PATH PLANNING MET- HOD FOR SWARMS OF UAVS.}

\begin{abstract}
Advances in robotics have resulted in the appearance of cooperative and autonomous use of different types of vehicles, in order to complete different types of work more efficiently. One of the fundamental aspects that allows to achieve this collaboration is the planning of safe routes, in such a way that it is possible to guarantee the free movement of collisions of each one of the vehicles by the work area. For this reason, in this work, it is proposed to plan multiple routes for a swarm of Unmanned Aerial Vehicles based on the creation of Probabilistic Road Maps. The objective is the implementation of a method that allows the construction of safe trajectories for each one of the agents of the swarm when a joint objective is desired, a labeled objective or when there are several objectives to be
\end{abstract}

completed and several vehicles are available to do so ( unlabeled), demonstrating that probabilistic roadmaps constitute a fast and effective method for the establishment of multiple routes. In addition, it is proposed to integrate this solution in an ROS architecture that allows, in the first instance, to obtain results within the Gazebo simulation environment and, in the future, a simple implementation of the algorithms presented in a real fleet of vehicles. The results obtained show optimum performance in terms of computing time for the different situations proposed and possible integration into a versatile framework such as $R O S$.

Keywords: UAVs · Planning · Swarms · Autonomous $\cdot$ Navigation $\cdot$ ROS.

\section{Referencias}

[1] Al-Kaff, A., Moreno, F. M., de la Escalera, A., \& Armingol, J. M. (2017, October). Intelligent vehicle for search, rescue and transportation purposes. In 2017 IEEE International Symposium on Safety, Security and Rescue Robotics (SSRR) (pp. 110-115). IEEE.

[2] Al-Kaff, A., Moreno, F. M., San José, L. J., García, F., Martín, D., de la Escalera, A., ... \& Garcéa, J. L. M. (2017, April). VBII-UAV: vision-based infrastructure inspection-UAV. In World Conference on Information Systems and Technologies (pp. 221-231). Springer, Cham.

[3] Claesson, A., Fredman, D., Svensson, L., Ringh, M., Hollenberg, J., Nordberg, P., ... \& Ban, Y. (2016). Unmanned aerial vehicles (drones) in out-of-hospital-cardiacarrest. Scandinavian journal of trauma, resuscitation and emergency medicine, 24(1), 124.

[4] Farooq, M. U., Ziyang, Z., \& Ejaz, M. (2017, December). Quadrotor UAVs Flying Formation Reconfiguration with Collision Avoidance Using Probabilistic Roadmap Algorithm. 
In 2017 International Conference on Computer Systems, Electronics and Control (ICCSEC) (pp. 866-870). IEEE.

[5] Faust, A., Oslund, K., Ramirez, O., Francis, A., Tapia, L., Fiser, M., \& Davidson, J. (2018, May). PRM-RL: Long-range robotic navigation tasks by combining reinforcement learning and sampling-based planning. In 2018 IEEE International Conference on Robotics and Automation (ICRA) (pp. 51135120). IEEE.

[6] Goerzen, C., Kong, Z., \& Mettler, B. (2010). A survey of motion planning algorithms from the perspective of autonomous UAV guidance. Journal of Intelligent and Robotic Systems, 57(1-4), 65.

[7] Kanistras, K., Martins, G., Rutherford, M. J., \& Valavanis, K. P. (2015). Survey of unmanned aerial vehicles (UAVs) for traffic monitoring. Handbook of unmanned aerial vehicles, 2643-2666.

[8] Koenig, N., \& Howard, A. (2004, September). Design and use paradigms for gazebo, an open-source multi-robot simulator. In 2004 IEEE/RSJ International Conference on Intelligent Robots and Systems (IROS)(IEEE Cat. No. 04CH37566) (Vol. 3, pp. 2149-2154). IEEE.

[9] Kuhn, H. W. (1955). The Hungarian method for the assignment problem. Naval research logistics quarterly, 2(1-2), 83-97.

[10] LaValle, S. M. (2006). Planning algorithms. Cambridge university press.

[11] Li, Q., Wei, C., Wu, J., \& Zhu, X. (2014, August). Improved PRM method of low altitude penetration trajectory planning for UAVs. In Proceedings of 2014 IEEE Chinese Guidance, Navigation and Control Conference (pp. 2651-2656). IEEE.

[12] Ma, K. C., Ma, Z., Liu, L., \& Sukhatme, G. S. (2018). Multi-robot informative and adaptive planning for persistent environmental monitoring. In Distributed Autonomous Robotic Systems (pp. 285-298). Springer, Cham.

[13] Meyer, J. (2014). Hector quadrotor ROS package website. www., Available: http://wiki. ros. org/hector_quadrotor.

[14] Quigley, M., Conley, K., Gerkey, B., Faust, J., Foote, T., Leibs, J., ... \& Ng, A. Y. (2009, May). ROS: an open-source Robot Operating System. In ICRA workshop on open source software (Vol. 3, No. 3.2, p. 5).
[15] Russell, S. J., \& Norvig, P. (2016). Artificial intelligence: a modern approach. Malaysia; Pearson Education Limited,.

[16] Samaniego, F., Sanchis, J., García-Nieto, S., \& Simarro, R. (2017, October). UAV motion planning and obstacle avoidance based on adaptive 3D cell decomposition: Continuous space vs discrete space. In 2017 IEEE Second Ecuador Technical Chapters Meeting (ETCM) (pp. 1-6). IEEE.

[17] Xue, Q., Cheng, P., \& Cheng, N. (2014, August). Offline path planning and online replanning of UAVs in complex terrain. In Proceedings of 2014 IEEE Chinese Guidance, Navigation and Control Conference (pp. 22872292). IEEE.

[18] Yan, F., Liu, Y. S., \& Xiao, J. Z. (2013). Path planning in complex $3 \mathrm{D}$ environments using a probabilistic roadmap method. International Journal of Automation and computing, 10(6), 525-533.

[19] Yan, Z., Jouandeau, N., \& Cherif, A. A. (2013). A survey and analysis of multi-robot coordination. International Journal of Advanced Robotic Systems, 10(12), 399.

[20] Yang, L., Qi, J., Xiao, J., \& Yong, X. (2014, June). A literature review of UAV 3D path planning. In Proceeding of the 11th World Congress on Intelligent Control and Automation (pp. 2376-2381). IEEE.

[21] Young, S., \& Kott, A. (2016). A survey of research on control of teams of small robots in military operations. arXiv preprint arXiv:1606.01288.

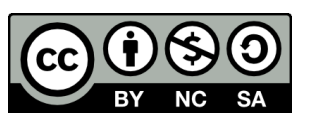
(C) 2019 by the authors. Submitted for possible open access publication under the terms and conditions of the Creative Commons Attribution CC BY-NC-SA 4.0 license (https://creativecommons.org/licenses/by-ncsa/4.0/deed.es). 